\title{
Optical Coherence Tomography as a Useful Adjunct for Diagnosis and Management of Acute Zonal Occult Outer Retinopathy
}

\author{
Lik Thai Lim, Vikas Shankar, Robert Blum \\ Tennent Institute of Ophthalmology, Gartnavel General Hospital, Great Western Road, \\ Glasgow, UK
}

\begin{abstract}
Acute zonal occult outer retinopathy is a rare condition that has traditionally been diagnosed by perimetry and electroretinogram. This report is of a 48-year-old man with acute zonal occult outer retinopathy, for which optical coherence tomography aided the diagnosis. Optical coherence tomography can be a useful adjunct for diagnosis and management of acute zonal occult outer retinopathy.
\end{abstract}

Key words: Electroretinography, Retinal diseases, Tomography, optical coherence, Visual field tests

Asian J Ophthalmol. 2011;12:181-2.

\section{Introduction}

Acute zonal occult outer retinopathy (AZOOR) is an uncommon condition that has traditionally been diagnosed with the aid of perimetry and electroretinography (ERG). This report is of a man with AZOOR, for which optical coherence tomography (OCT) aided the diagnosis, and shows how OCT can be a useful adjunct for the diagnosis and management.

\section{Case Report}

A 48-year-old previously healthy man presented in 2007 with a 1-week history of photopsia and floaters in the left eye. His bestcorrected visual acuity was $6 / 4$ on the Snellen chart for both eyes.

Ocular examination of the anterior and posterior segments was unremarkable, and included a healthy looking fundus in both eyes. Multifocal ERG showed flattened responses in the retinal areas temporal to the fovea in the left eye. Static perimetry revealed a corresponding nasal visual field defect in the left eye. Based on the history, ocular examination, perimetry, and ERG findings, a diagnosis of AZOOR was made. This diagnosis was confirmed by OCT, which showed absence of the photoreceptor layer temporal to the fovea in the left eye, coinciding with the corresponding areas where flattened ERG responses were documented (Figure 1).

The patient was treated conservatively and was reviewed after 4 months. He reported complete spontaneous resolution of the

Correspondence: Dr Lik Thai Lim, Tennent Institute of Ophthalmology, Gartnavel General Hospital, Glasgow G12 OYN, UK.

Tel: (44 78) 4361 7788;

E-mail: likthai@doctors.org.uk
Figure 1. Optical coherence tomography showing absence of the photoreceptor layer temporal to the fovea in the left eye.

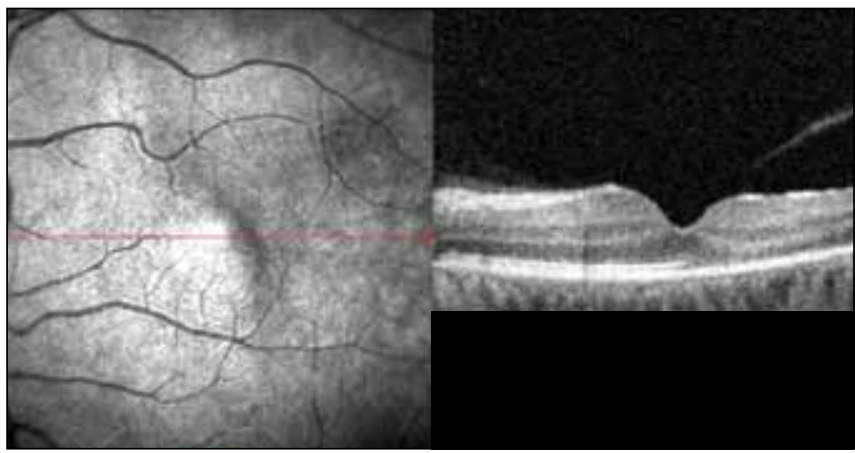

Figure 2. Optical coherence tomography showing regeneration of the photoreceptor layer temporal to the fovea in the left eye.

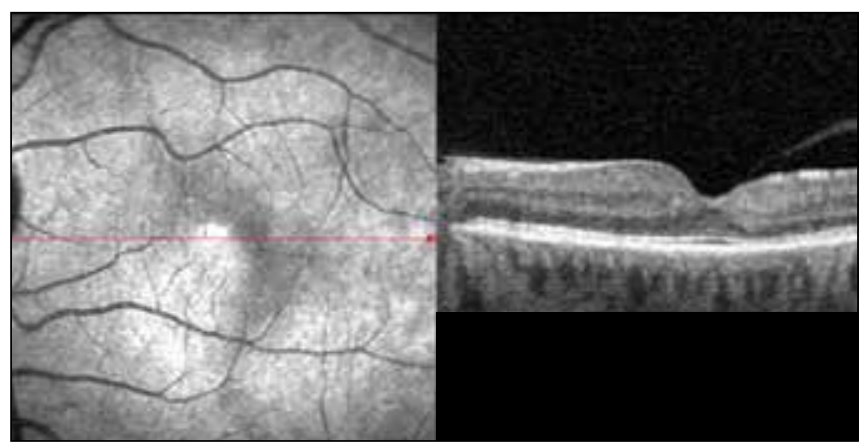

ocular symptoms. Subsequent OCT confirmed the resolution of AZOOR, demonstrating the presence of the photoreceptor layer in the corresponding areas (Figure 2). Subsequent visual field tests and ERG were within normal limits. The patient was discharged from the clinic. 


\section{Discussion}

AZOOR is a rare ocular condition, which can present with a wide variety of ocular symptoms, including reduced vision, photopsia, floaters, and visual field defects. The condition is due to acute retinal photoreceptor pathology of unknown cause, but it has a good prognosis.

AZOOR is traditionally diagnosed by the ophthalmic history, ocular examination, perimetry, and ERG. ${ }^{1,2}$ Ocular examination can be unremarkable in some patients, therefore, further investigations may be required, namely perimetry and ERG.$^{1,2}$ However, with advancements in technology, OCT can be used to clearly demonstrate the pathology, showing absence of the photoreceptor layer in the acute phase (Figure 1), and regeneration of the photoreceptor layer in the resolution phase (Figure 2). This feature is in keeping with AZOOR, as described in a recent study by Takai et al. ${ }^{3}$
Patients with AZOOR can expect some improvement of visual symptoms after the initial presentation, with most experiencing near complete resolution. ${ }^{1,2}$ However, for this patient, resolution was complete, with total resolution of visual symptoms and OCT evidence of a fully regenerated photoreceptor layer. Therefore, OCT can be a useful adjunct to the diagnosis of AZOOR and can be used to monitor the progress of such patients.

\section{References}

1. Gass JD, Agarwal A, Scott IU. Acute zonal occult outer retinopathy: a long-term follow-up study. Am J Ophthalmol. 2002;34:329-39.

2. Francis PJ, Marinescu A, Fitzke FW. AZOOR: towards a set of diagnostic criteria. Br J Ophthalmol. 2005;89:70-3.

3. Takai $Y$, Ishiko S, Kagokawa $H$, Fukui K, Takahashi A, Yoshida A. Morphological study of acute occult outer retinopathy (AZOOR) by multiplanar optical coherence tomography. Acta Ophthalmol. 2009;87:408-18. 